Sisto, M., Pérez-Fuentes, M.C., Gázquez-Linares, J.J. \& Molero-Jurado, M.M. (2021). Actitudes hacia la inclusión educativa de alumnos con discapacidad: variables relativas al profesorado y a la organización escolar en Educación Primaria. Revista Electrónica Interuniversitaria de Formación del Profesorado, 24(1), 221-237.

DOI: https://doi.org/10.6018/reifop.397841

\title{
Actitudes hacia la inclusión educativa de alumnos con discapacidad: variables relativas al profesorado y a la organización escolar en Educación Primaria
}

Maria Sisto, María del Carmen Pérez-Fuentes, José Jesús Gázquez-Linares, María del Mar Molero-Jurado

Universidad de Almería

\section{Resumen}

La falta de colaboración entre profesionales de la educación influye en el desarrollo de actitudes, especialmente negativas, que se convierten en las barreras que mayormente inciden en el proceso de inclusión educativa. El objetivo de este trabajo fue analizar la relación entre actitudes hacia la inclusión de alumnos con discapacidad y algunas variables relacionadas con la organización escolar y con el profesorado ( $\left.N=102 ; M_{\text {edad }}=43,49 ; D T=10,6\right)$ de doce centros de educación primaria, tanto públicos como concertados. Se empleó una adaptación de la escala «Opinions Relative to Integration of Students with Disabilities» (Antonak y Larrive, 1995) a partir de algunas traducciones al castellano (García y Alonso, 1985; Mula et al., 2002; Sales, Moliner, y Sanchiz, 2001; Tárraga, Grau, y Peirat, 2013). Los resultados destacan actitudes negativas en el profesorado cuando hay carencia de recursos y cuando la responsabilidad de la elaboración, puesta en práctica y seguimiento de las adaptaciones curriculares no está compartida entre diferentes profesionales. Se plantea la importancia de encargarse a nivel administrativo de la formación activa de los profesionales educativos, independientemente de sus especialidades, para que trabajen de forma colaborativa proporcionando medidas educativas adecuadas para todo el alumnado.

\section{Palabras clave}

Inclusión educativa; profesorado; actitudes; educación primaria.

\section{Contacto:}

Maria Sisto, Departamento de Psicología, Edificio Departamental de Humanidades y CC.EE-I, La Cañada de San Urbano, 04120, Almería. Email: ms168@ual.es. 


\title{
Attitudes to inclusion of students with disability: variables related of teachers and organization in the primary school
}

\begin{abstract}
The lack of collaboration between education professionals influences the development of negative attitudes, which become the barriers that mostly condition the educational inclusion process. The objectives of this article are to analyse the relationship between inclusive attitudes to students with disability and some variables related to organization and teachers ( $N=102 M=43.49 ; S D=10.6)$ of twelve primary education centers, both public and private. An adaptation of the "Opinions Relative to Integration of Students with Disabilities" scale (Antonak and Larrive, 1995) was used, based on some Spanish translations (García and Alonso, 1985; Mula et al., 2002; Sales, Moliner, and Sanchiz, 2001; Tárraga, Grau, and Peirat, 2013). The results highlight negative attitudes in teachers when there is a lack of resources, specifically when the responsibility for the development, implementation and monitoring of curricular adaptations is not shared between different professionals. It is suggested the importance of taking care of, in an administrative level, the active training of educational professionals, regardless of their specialties, in order to work in a collaboratively way providing appropriate educational measures for all students.
\end{abstract}

\section{Key words}

Educational inclusion; teachers; attitudes; primary education.

\section{Introducción}

\section{La Educación Inclusiva en España}

La perspectiva de la Educación Inclusiva (EI) es un recorrido complejo que implica presupuestos epistemológicos, culturales, éticos, políticos y sociales, y que presupone un cambio en la cultura, organización y prácticas de los centros, para atender a las necesidades educativas de todo el alumnado. La enseñanza basada en la El se adapta al estudiante, mediante acciones dirigidas a eliminar o minimizar las barreras físicas, personales o institucionales y, así, garantizar el acceso, la participación en las actividades formativas, e iguales oportunidades de aprendizaje para cada alumno, sean cuales sean sus características personales, psicológicas, sociales (Canevaro y Malaguti, 2015; Echeíta, 2016). Los últimos treinta años han sido significativos a nivel histórico-legislativo para la evolución del concepto de El. No obstante, la situación española refleja cierta complejidad: persisten grandes dificultades para generalizar la inclusión en el ámbito educativo, cómo queda confirmado por el Informe del Comité Español de Representantes de Personas con Discapacidad (CERMI, 2010). La European Agency for Development in Special Needs Education, que regularmente recoge datos sobre las dinámicas educativas inclusivas, destaca que un $2,3 \%$ de alumnos con discapacidad sigue cursando sus estudios aisladamente, específicamente en la etapa de educación obligatoria, bien en centros de Educación Especial o bien en aulas específicas ubicadas en colegios ordinarios (Canevaro y Malaguti, 2015; Florian, 2014). Estos datos sugieren algunas criticidades que impiden a los centros educativos ordinarios españoles incluir a todos los estudiantes con discapacidad. Sin embargo, en aquellos países cuyos gobiernos cerraron los centros de educación 
especial, integrando a todos los alumnos en colegios ordinarios, no hubo secuelas negativas irreversibles para el sistema educativo (Piccioli, 2019; Rajakaltio y Mäkinen, 2014).

\section{Educación Inclusiva y variables relacionadas con el profesorado y la organización escolar}

Los resultados de diferentes estudios indican que las actitudes del profesorado intervienen en el desarrollo del autoconcepto de los alumnos con discapacidad, influenciando el proceso de socialización e inclusión de los mismos. Las actitudes, especialmente si son negativas, representan las barreras mentales que mayormente obstaculizan la participación de estos alumnos (Flórez, León, y Alcedo, 2009; Martínez, 2012). Todavía no se ha logrado un acuerdo respecto a la causa de actitudes discriminatorias, pero resulta importante profundizar en el tipo de disposición adoptado por los profesionales educativos y en los factores responsables de una educación inclusiva eficaz, puesto que incluso la aceptación de principios inclusivos por parte de estos profesionales no siempre corresponde directamente a la puesta en marcha de prácticas inclusivas (García, Inglés, Vicent, González, y Mañas, 2013). Entre los factores implicados en la postura del profesorado ante la inclusión, los estudios nacionales e internacionales, detectan los siguientes:

-Años de docencia, género y edad. Kalyva, Gojkovic, y Tsakiris (2007) apuntan que los docentes que llevan menos años de experiencia en la enseñanza apoyan más la inclusión; sin embargo, otros investigadores han obtenido hallazgos inconsistentes con respecto a esta variable (Koay, Lim, Sim, y Elkins, 2006; Van Reusen et al., 2001). Además, Batsiou, Bebetsos, Panteli, y Antoniou (2008) han demostrado que son los varones quienes muestran posturas inclusivas más positivas, mientras que en el estudio Chiner (2011) se han observado mejores actitudes en las mujeres. Incluso existen estudios que no han hallado ninguna diferencia estadísticamente significativa analizando la variable género (De Almeida y Castiñeiras, 2009; Gónzalez-Gil, Martín-Pastor, Poy, y Jenaro, 2016). Así mismo, algunas investigaciones destacan relaciones estadísticamente significativas entre edad y actitudes inclusivas del profesorado: Tortello y Elgier (2014) confirmaron que la edad correlaciona negativamente con las actitudes favorables. No obstante, los resultados existentes acerca del peso de estos factores son contrapuestos (Alghazo y Gaad, 2004; Ernst y Rogers, 2009; Forlin, Loreman, Sharma, y Earle, 2009; Gómez, 2016; Koay et al., 2006).

-Competencia profesional, formación y nivel educativo. Se ha puesto de manifiesto que un mayor sentimiento de competencia profesional influye de forma positiva en el desempeño del profesorado en llevar a cabo una educación inclusiva (De Boer et al., 2011; Hsien, 2007) $y$, también, que el profesorado con carencias formativas desarrolla actitudes menos propicias hacia la inclusión en centros ordinarios, respecto al profesorado con formación previa (García, 2016; Tárraga, Grau, y Peirat, 2013). No obstante, Gallego y Rodríguez (2012) y Wilkins y Nietfeld (2004) hallaron resultados inconsistentes con respecto a la variable formación. Otros investigadores detectaron una relación significativa entre el nivel educativo y la postura del profesorado ante la inclusión: se descubrió que los participantes con un mayor nivel, es decir, aquellos que poseían títulos de posgrado, tenían actitudes significativamente más positivas en comparación con sus compañeros que no poseían títulos de posgrado (Dupoux, Wolman, y Estrada, 2005; Sharma, Moore, y Sonawane, 2009). Sin embargo, otros trabajos encontraron hallazgos inconsistentes analizando esta variable (DeSimone y Parmar, 2006; Sharma, Forlin, Loreman, y Earle, 2006).

-Recursos disponibles, responsabilidad compartida y colaboración entre profesionales. La inclusión de los estudiantes con discapacidad se ve favorecida por la presencia de recursos en los colegios y de otros apoyos para la docencia, así como por la disminución de la ratio docente/alumno; la falta de estos elementos coincide con la creación de barreras que impiden realizar procesos inclusivos, pudiendo desembocar en exclusión educativa 
(Gandasegui y Méndez, 2014; Jordan, Schwartz, y McGhie, 2009). Atendiendo a la responsabilidad del profesorado hacia el alumnado con discapacidad, la literatura científica informa que en España las prácticas de educación compensatoria y atención a la diversidad no se contemplan como responsabilidad compartida entre todos los profesores, sino como responsabilidad prioritaria del profesor especialista, una figura profesional que, en ocasiones, ha sido muy enfatizada (Sandoval, Simón, y Echeíta, 2012). Ya ha sido demostrado que la carga adicional de responsabilidad percibida por el profesorado, cuando en una clase ordinaria está incluido un alumno con discapacidad, influye negativamente en sus actitudes inclusivas (Kavale y Forness, 2000; Tam, Seevers, Gardner, y Heng, 2006). Diferentes estudios destacan que los profesores de área entienden las adaptaciones curriculares como programas paralelos a la programación del aula, prefiriendo que sea exclusivamente el maestro especialista quien se responsabilice y encargue de atender a las diversidades de los estudiantes, trabajando individualmente o con pequeños grupos, aunque, en ambos casos, fuera de la clase (Carreres y Arnaiz, 2010; Marchesi, Durán, Giné, y Hernández, 2009). De acuerdo con la legislación nacional y la normatividad propia de cada comunidad autónoma, las direcciones gubernamentales educativas se encargan de proporcionar los apoyos y recursos para la inclusión haciendo referencia a tres perfiles profesionales del profesorado (Pedagogía Terapéutica; Audición y Lenguaje; Compensatoria) con funciones de coordinación-asesoramiento de los apoyos y de realización-adaptación curricular para el alumnado con discapacidad. Sin embargo, cada comunidad autónoma establece de forma independiente las funciones del profesor especialista (Ley Orgánica 2/2006). Concretamente, en Andalucía, son los profesores de Pedagogía Terapéutica quienes tienen la responsabilidad de realizar las adaptaciones curriculares para el alumnado con discapacidad en colaboración con los profesores de áreas, y no al revés, como informa el estudio de Moya y Díez (2012). Otro estudio llevado a cabo en Jaén, revela que la mayoría de los profesores manifiestan que no se produce colaboración con otros profesionales distintos a los del centro y a los equipos de orientación en su labor tutorial (Torres y Fernández, 2015).

El asunto de la atención a la diversidad, y la oportunidad de ofrecer medidas educativas inclusivas al interior de las instituciones educativas, queda pendiente por resolver, como se evidencia revisando la literatura sobre el tema (Flores y Gallego, 2015; Tárraga, Grau, y Peirat, 2013). Además, disponemos de datos discrepantes en orden a la postura de los profesionales de la educación ante la inclusión de alumnos con discapacidad: de una parte, se han destacado actitudes neutrales o negativas (De Boer, Pijil, y Minnaert, 2011; Hwang y Evans, 2011); por otra parte, se han señalado actitudes positivas (Avramidis, Bayliss, y Burden, 2000; Smith, 2004).

\section{Hipótesis y objetivos}

El hecho de que no se haya profundizado el estudio de determinadas variables de profesores y centros con respecto a la El, conduce a considerar la importancia de seguir investigando en este ámbito. Para resolver la ambivalencia acerca de las actitudes inclusivas del profesorado, planteamos la importancia de investigar la influencia de características específicas implicadas en el desarrollo de estas actitudes. Por lo tanto, el presente estudio tiene como objetivo general aportar nuevos datos a la investigación sobre la inclusión educativa en centros ordinarios. Para alcanzarlo, partiremos de los siguientes objetivos específicos: a) analizar variables relacionadas con la organización escolar de centros tanto públicos como concertados (frecuencia y tipo de discapacidad de los alumnos incluidos, presencia de recursos, ratio profesor especialista/alumnado con discapacidad) que puedan influir en las actitudes inclusivas; b) comprobar la relación entre algunas variables del profesorado y la postura adoptada ante la inclusión. 
A partir de la evidencia empírica previa, se plantean las siguientes hipótesis: 1) existe una situación de desigualdad entre centros públicos y concertados en orden a la frecuencia-tipo de diagnóstico del alumnado con discapacidad y a los recursos presentes; 2) los profesionales que llevan menos años de docencia presentarían una postura menos negativa hacia la inclusión; $y, 3$ ) existe una relación significativa inversa entre la postura positiva del profesorado y la creencia de que la responsabilidad de las adaptaciones curriculares sea exclusiva de los especialistas.

\section{Método}

Se ha llevado a cabo un diseño de investigación descriptivo, correlacional y transversal.

\section{Participantes}

A partir de una población de 134 centros que comparten las mismas características en Almería capital (públicos por un lado y concertados por otro), con registro en la Consejería de Educación de la Junta de Andalucía, fueron seleccionados 12 centros de Educación Primaria ( 5 públicos y 7 concertados) que presentaban el criterio de selección, es decir, incluir a alumnos con alguna discapacidad diagnosticada y que contaban con profesores especialistas en Pedagogía Terapéutica.

Del total de la muestra, que asciende a 102 profesionales de la educación, 31 son varones $(30,4 \%)$ y 71 mujeres (69,6\%). De ellos, el $59,8 \%$ tiene una edad que oscila entre 39 y 58 años, el $28,4 \%$ entre 18 y 38 años y el $11,8 \%$ entre 59 y 78 , con un valor mínimo de 23 años y un valor

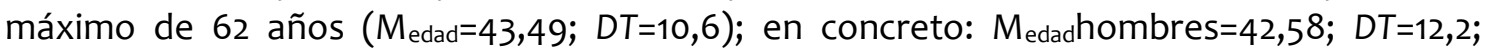
$M_{\text {edad }}$ mujeres=43,88; $D T=9,9$. Atendiendo al nivel educativo, el $47,1 \%$ de profesionales posee un título de grado; el 21,6\% otro título (Conservatorio o especialidades), el 15,7\% un Máster, el 10,8\% ha llegado hasta la etapa de la educación secundaria posobligatoria (Módulos de Formación Profesional/Plan de estudios con carácter profesionalizador) y el $4,9 \%$ ha conseguido un Doctorado. Por último, los profesores especialistas en Pedagogía Terapéutica (de apoyo para la inclusión) se distribuyen de la siguiente forma: el $20 \%$ desempeña su labor en centros públicos y el $80 \%$ en centros concertados.

\section{Instrumentos}

El cuestionario administrado al profesorado consta de dos partes. La primera recoge variables demográficas y de carácter descriptivo: sexo, edad, nivel educativo, años/especialidad de docencia, tipo de centro/curso en el que se imparte la docencia, número de alumnos con discapacidad incluidos en el centro, tipo y grado de discapacidad diagnosticada de los mismos. Para la segunda parte se empleó una versión de la escala Opinions Relative to Integration of Students with Disabilities (ORI) de Antonak y Larrivee (1995), utilizada en España gracias a las adaptaciones traducidas por García y Alonso (1985), Mula et al. (2002), Sales, Moliner, y Sanchiz (2001) y Tárraga, Grau, y Peirat (2013). La escala consta de 25 ítems de tipo Likert con 5 opciones de respuesta: Muy en Desacuerdo-MD; En Desacuerdo-ED; De Acuerdo-DA; Muy de Acuerdo-MA; Indiferente/No sé-NS. Su consistencia interna, así como la de las tres sub-escalas extraídas (Actitudes-l; Aspectos Centro Educativo; Actitudes-II) ha sido previamente demostrada con valores entre $\alpha=.55 \div \alpha=.83$ (Martínez y Castillo, 2007; Mula et al., 2002; Tárraga, Grau, y Peirat, 2013).

En el presente estudio la consistencia interna de la escala completa queda demostrada con la estimación de los coeficientes ordinales $a l f a=.80$ y theta $=.83$. La primera escala, con un alfa ordinal de .82, da lugar a dos factores de primer orden (Actitudes Favorables/Actitudes Desfavorables); la segunda, con un alfa ordinal de .75, a dos factores (Recursos 
Centros/Dotación Espacios) y la tercera, con un alfa ordinal de .76, a tres factores (Inclusión Positiva/Inclusión Negativa Aula Ordinaria/Inclusión Negativa para Alumnos). Por último, de la matriz factorial rotada se obtienen dos dimensiones de segundo orden: Postura General Negativa y Postura General Positiva.

\section{Procedimiento}

El presente trabajo fue elaborado entre los años 2015-2017. En principio, se revisó el estado de la cuestión relativo a la El dentro del sistema educativo español en comparación con otros países europeos y las variables implicadas en las dinámicas inclusivas de los centros educativos ordinarios. Después de delimitar los objetivos e hipótesis del trabajo, así como la muestra y los instrumentos, se solicitó un permiso a la Delegación Provincial de Almería para acceder a los centros. Sucesivamente se accedió a los colegios para indicar el objetivo de la investigación a los directores, profesores, y a las familias del alumnado. Tras la distribución de los cuestionarios, en algunos colegios se cumplimentaron y recogieron en el mismo día durante un claustro de profesores, mientras que en otros centros fueron los directores quienes distribuyeron los cuestionarios y se encargaron de recogerlos y guardarlos en un sobre, para su posterior recogida, análisis e interpretación de los resultados.

\section{Análisis de datos}

Para analizar los resultados se empleó el paquete estadístico SPSS versión 15.0 para Windows. Para examinar la fiabilidad de la escala utilizada para la recogida de datos: 1) Primero, se lleva a cabo un análisis factorial exploratorio en la matriz de correlación policórica, utilizando el software FACTOR (Lorenzo-Seva y Ferrando, 2006). Los datos se calculan bajo un criterio de análisis paramétrico y rotación promin. 2) Para calcular los coeficientes ordinales alfa y theta, se utiliza la hoja de cálculo Excel desarrollada por Domínguez-Lara (2018), con un cómputo basado en los datos del análisis de correlación policórica y, por lo tanto, más adecuado para calcular el fiabilidad de escalas con respuesta ordinal o de escala tipo Likert (Elosua y Zumbo, 2008).

Por otro lado, se llevó a cabo un análisis descriptivo, sometiendo los datos a un tratamiento estadístico sobre frecuencia, medias y desviación. También, se emplearon análisis factoriales exploratorios con el método de extracción de componentes-rotación Varimax, para integrar las respuestas en diferentes factores y conocer posibles correlaciones entre ellos.

Además, se realizó la técnica de correlación bivariada $r$ de Pearson, basada en la asociación lineal, para verificar la dirección/intensidad entre las variables de análisis posteriores, así como se llevaron a cabo análisis de regresión lineal múltiple para identificar las relaciones causales entre las variables dependientes e independientes. Por último, la Prueba $t$ de Student para muestras independientes y la Prueba Anova de un factor, sirvieron para conocer la relación estadística entre las variables predictoras y la postura tanto positiva como negativa del profesorado ante la inclusión.

\section{Resultados}

Atendiendo a los factores relacionadas con la organización escolar, en el gráfico 1 se muestran los datos que hacen referencia a la ratio profesor especialista/alumnado con discapacidad, en ambos centros educativos (1:3 en los públicos; 1:4 en los concertados). 
Por otro lado, en la tabla 1, se observa cómo se distribuyen los alumnos con discapacidad matriculados, en relación con el tipo de discapacidad diagnosticada y el tipo de colegio en el que están incluidos.

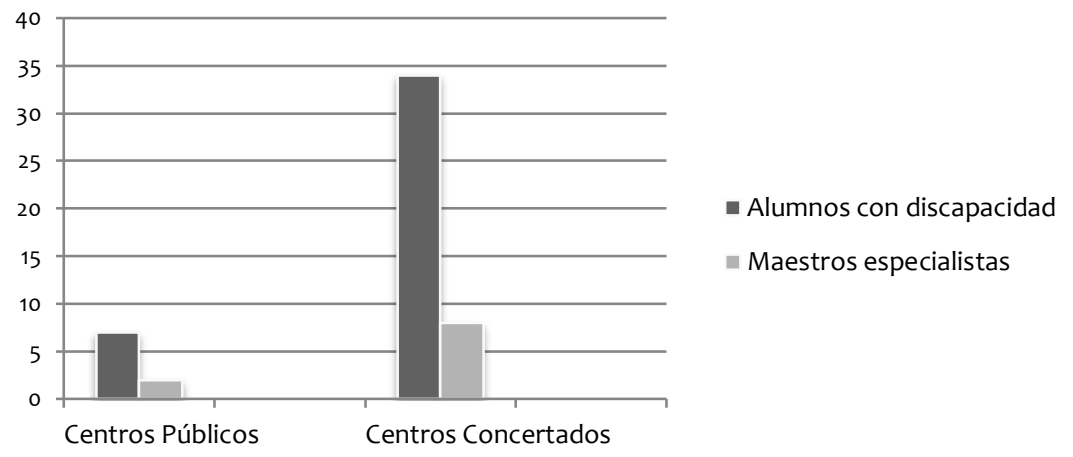

Gráfico 1.

Distribución de alumnado con discapacidad y profesorado especialista, según tipo de centro

Tabla 1.

Distribución de alumnos con discapacidad según grado de discapacidad y tipo de centro

\begin{tabular}{lcc}
\hline \multirow{2}{*}{ Tipo de discapacidad (grado) } & \multicolumn{2}{c}{ Tipo de Centro } \\
\cline { 2 - 3 } & Público & Concertado \\
\hline Ligero (5-24\%) & $72.2 \%$ & $100 \%$ \\
\hline Moderado (25-49\%) & $27.8 \%$ & 0 \\
\hline Grave (50-95\%) & 0 & 0 \\
\hline Completo (100\%) & 0 & 0 \\
\hline
\end{tabular}

Por otro lado, en la tabla 2 se someten a una Prueba $t$ los factores de primer orden "dotación espacios"|"recursos centro" (relacionados con la organización escolar) con la variable predictora "tipo de centro", en la que no se obtienen diferencias estadísticamente significativas en la respuesta sobre la dotación de espacios, pero sí, en la respuesta sobre la presencia de recursos para la inclusión $(t=1.98 ; p<.01)$. En efecto, los profesores de los centros concertados obtienen mayor puntuación $(M=.14 ; D T=1.02)$ en comparación con los del público $(M=-.26 ; D T=1.01)$, acerca de la presencia en sus centros de recursos y apoyos a la docencia para atender a la discapacidad.

Tabla 2.

Recursos centro y dotación espacios según tipo de centro. Descriptivos y Pruebat

\begin{tabular}{|c|c|c|c|c|c|c|c|c|}
\hline \multirow{3}{*}{ Escala } & \multicolumn{6}{|c|}{ Tipo de Centro } & \multirow{3}{*}{$t$} & \multirow{3}{*}{$p$} \\
\hline & \multicolumn{3}{|c|}{ Público } & \multicolumn{3}{|c|}{ Concertado } & & \\
\hline & $N$ & Media & DT & $N$ & Media & DT & & \\
\hline Recursos centro & 36 & -.26 & 0.92 & 66 & .14 & 1.02 & $-1.98 *$ & .04 \\
\hline Dotación espacios & 36 & -.04 & .99 & 66 & .02 & 1.01 & -.27 & .79 \\
\hline
\end{tabular}

Nota: *La relación es significante al nivel 0.05 
En la tabla 3 se muestra la matriz de correlaciones de las variables latentes obtenidas sometiendo los factores de primer orden a un análisis de correlación $r$ de Pearson. Se aprecia la existencia de una relación positiva estadísticamente significativa entre las variables «Actitud Favorable» e «Inclusión Positiva» con un coeficiente $r .48$ ( $p=.000)$ lo que indica co-variabilidad entre las dos variables. Por otro lado, la variable «Actitud Desfavorable» correlaciona de manera positiva con la variable «Inclusión Negativa Aula Ordinaria», como demuestra el coeficiente de significación $r .45(p=.000)$, mientras que la variable «Recursos Centro» se encuentra relacionada de modo positivo con las dos variables «Actitud Favorable» y «Actitud Desfavorable». Además, tras utilizar el método de rotación Varimax, se observa la nulidad de los coeficientes $r$ de los factores provenientes del mismo análisis factorial: «Actitud Favorable/«Actitud Desfavorable»; «Dotación Espacios»/«Recursos Centro»; «Inclusión Positiva»/«Inclusión Negativa Aula Ordinaria»/«Inclusión Negativa Alumnos». Por lo tanto, las variables latentes obtenidas son independientes entre sí, puesto que no hay relación ni asociación entre ellas (Tabla 3).

Tabla 3 .

Matriz de correlación r de Pearson entre los factores de primer orden

\begin{tabular}{|c|c|c|c|c|c|c|c|}
\hline & (1) & $(2)$ & (3) & (4) & (5) & $(6)$ & $(7)$ \\
\hline Actitud Favorable (1) & 1 & & & & & & \\
\hline Actitud Desfavorable (2) & .000 & 1 & & & & & \\
\hline Recursos centro (3) & $.204(*)$ & $.234\left(^{*}\right)$ & 1 & & & & \\
\hline Dotación espacios (4) & .035 & .051 & .000 & 1 & & & \\
\hline Inclusión positiva (5) & $.480(* *)$ & .084 & .159 & .118 & 1 & & \\
\hline Inclusión negativa aula ordinaria (6) & .049 & $.456(* *)$ & .103 & -.046 & .000 & 1 & \\
\hline Inclusión negativa alumnos (7) & -.032 & .192 & .093 & -.109 & .000 & .000 & 1 \\
\hline
\end{tabular}

Nota: *La correlación es significante al nivel 0.05 (bilateral). ** La correlación es significativa al nivel 0.01 (bilateral). $N=102$.

En la tabla 4, con el primer análisis de regresión lineal múltiple, se considera como Variable Dependiente (VD) el factor de segundo orden "postura general positiva" y como variables predictoras: género, edad, años de docencia, nivel educativo, tipo de centro, responsabilidad sobre discapacidad. Con el modelo jerárquico de regresión, los datos obtenidos (puntuaciones $p$ y coeficientes Anova) muestran 4 de las 6 variables independientes que explican el $32 \%$ de la varianza $\left(R^{2}=.32 ; F=7.416, g l=6 ; p=.000\right): 1$. «Edad»; 2. "La responsabilidad de las adaptaciones curriculares es de los psicopedagogos»; 3. «La responsabilidad de las adaptaciones curriculares es compartida entre especialistas y profesores de áreas»; y 4. «La formación sobre discapacidad responde más a una política común del centro que a iniciativas individuales del profesorado».

En concreto, para identificar cómo difieren entre sí los subconjuntos de las medias que se refieren a la variable edad se ha llevado a cabo una Prueba Anova con post-hoc (DMS). Los resultados obtenidos se muestran en la tabla $5(F=3.72 ; p<.05)$ y evidencian que la diferencia en la postura general positiva se da únicamente entre el grupo g1 (profesorado con edad entre 18 y 38 años) y los demás (39-58 años; 59-78 años), puesto que no se observa entre g2-g3.

En la tabla 6, se muestra el segundo análisis de regresión lineal múltiple entre el factor dependiente de segundo orden "postura general negativa" y cuatro variables predictoras: género, años de docencia, nivel educativo, y tipo de centro. No se dan diferencias estadísticamente significativas respecto al género, $(t=76.41 ; p=.28)$, al tipo de centro y al nivel educativo $(t=70.96 ; p=.49)$. 
Tabla 4 .

Análisis de regresión múltiple con la VD de segundo orden "postura general positiva"

\begin{tabular}{lccccc}
\hline & \multicolumn{2}{c}{$\begin{array}{c}\text { Coeficientes No } \\
\text { Estand. }\end{array}$} & $\begin{array}{c}\text { Coeficientes } \\
\text { Estand. }\end{array}$ & t & Sig. \\
\cline { 2 - 6 } & B & Error típ. & Beta & & \\
\hline Constante) & -.996 & 416 & & -2.393 & .019 \\
\hline Edad & -.414 & .141 & -.254 & -2.939 & $.004^{*}$ \\
\hline Responsabilidad...especialistas & .048 & .086 & .052 & .557 & .579 \\
\hline Responsabilidad... psicopedagogos & -.224 & .071 & -.290 & -3.171 & $.002^{*}$ \\
\hline Responsabilidad... tutores & .019 & .077 & .024 & .243 & .809 \\
\hline Responsabilidad... compartida & .404 & .114 & .321 & 3.551 & $.001^{*}$ \\
\hline Formación.... iniciativas centro & .169 & .058 & .249 & 2.889 & $.005^{*}$ \\
\hline
\end{tabular}

Nota: *La relación es significante al nivel 0.05

Tabla 5 .

Edad y postura general positiva. Descriptivos y ANOVA (Post Hoc DSM)

\begin{tabular}{|c|c|c|c|c|c|c|c|}
\hline \multirow[t]{2}{*}{ Variable } & \multirow[t]{2}{*}{ Edad } & \multirow[t]{2}{*}{$N$} & \multirow[t]{2}{*}{ Media } & \multirow[t]{2}{*}{$\mathrm{DT}$} & \multicolumn{2}{|c|}{ ANOVA } & \multirow{2}{*}{$\begin{array}{c}\text { Diferencia de } \\
\text { medias (DMS) } \\
\left|g_{1-g 2}\right| *\end{array}$} \\
\hline & & & & & $F$ & Sig. & \\
\hline \multirow{3}{*}{$\begin{array}{l}\text { Postura general } \\
\text { positiva }\end{array}$} & 18-38 años (g1) & 29 & .39 & .46 & 3.72 & .03 & $|g 2-g 3|$ \\
\hline & 39-58 años (g2) & 61 & -.11 & 1.10 & & & $\mid$ g1-g3|* \\
\hline & 59-78 años (g3) & 12 & -.39 & 1.13 & & & \\
\hline
\end{tabular}

Nota: * La diferencia de media es significativa al nivel 0.05

Tabla 6.

Análisis de regresión múltiple con la VD de segundo orden «postura general negativa»

\begin{tabular}{lccccc}
\hline & \multicolumn{2}{c}{$\begin{array}{c}\text { Coeficientes No } \\
\text { Estand. }\end{array}$} & $\begin{array}{c}\text { Coeficientes } \\
\text { Estand. }\end{array}$ & t & Sig. \\
\cline { 2 - 6 } & $\mathrm{B}$ & Error típ. & Beta & & \\
\hline (Constante) & -.315 & .363 & & -.867 & .388 \\
\hline Género & .214 & .212 & .099 & 1.009 & .315 \\
\hline Años de docencia & .204 & .094 & .221 & 2.169 & $.033^{*}$ \\
\hline Nivel educativo & -.084 & .074 & -.112 & -1.132 & .260 \\
\hline Tipo de colegio & .220 & .210 & .105 & 1.046 & .298 \\
\hline
\end{tabular}

Nota: La relación es significante al nivel 0.05

Sin embargo, los resultados del Anova y el valor del estadístico de Prueba $F$ significativamente distinto de 1 para cualquier nivel de significación $(p<.05)$ indican que la variable años de docencia influye en la postura general negativa. Los resultados de la 
Prueba Post-Hoc (DMS) indican que existe una correlación positiva entre años de docencia y postura negativa, es decir, a medida que aumentan los años de docencia, se incrementa la postura general negativa del profesorado.

Específicamente, la diferencia estadísticamente significativa se da solamente entre el g4 (profesores que llevan 31 o más años de docencia) y todos los demás; en efecto, no se observa entre el g1-g2, ni entre el g1-g3, ni tampoco entre el g2-g3 (Tabla 7).

Tabla 7.

Años de docencia y postura general negativa. Descriptivos y ANOVA (Post Hoc DSM)

\begin{tabular}{|c|c|c|c|c|c|c|c|}
\hline \multirow[t]{2}{*}{ Variable } & \multirow[t]{2}{*}{ Años de docencia } & \multirow[t]{2}{*}{$N$} & \multirow[t]{2}{*}{ Media } & \multirow[t]{2}{*}{ DT } & \multicolumn{2}{|c|}{ ANOVA } & \multirow{2}{*}{$\begin{array}{c}\begin{array}{c}\text { Diferencia de medias } \\
\text { (DMS) }\end{array} \\
\text { g1-g2 }|| g 2-g_{3} \mid\end{array}$} \\
\hline & & & & & $F$ & Sig. & \\
\hline \multirow{4}{*}{$\begin{array}{l}\text { Postura general } \\
\text { negativa }\end{array}$} & 1-10 años (g1) & 31 & -.08 & .91 & \multirow[t]{4}{*}{3.27} & \multirow[t]{4}{*}{.02} & $\left|g_{3}-g_{4}\right| *\left|g_{1}-g_{3}\right|$ \\
\hline & 11-20 años (g2) & 31 & -.20 & 1.02 & & & $\left|g_{1-g}\right| *\left|g_{2}-g_{4}\right| *$ \\
\hline & 21-30 años (g3) & 22 & -.13 & 1.08 & & & \\
\hline & 31-jubilación (g4) & 18 & .64 & .78 & & & \\
\hline
\end{tabular}

Nota: * La diferencia de media es significativa al nivel 0.05

\section{Discusión}

El primero objetivo de este estudio fue analizar variables relacionadas con la organización escolar de centros ordinarios de educación primaria, tanto públicos como concertados: presencia de recursos humanos (profesorado especialista en pedagogía terapéutica) y materiales (apoyos a la docencia), frecuencia-tipo de discapacidad de alumnos incluidos.

En primer lugar, con respecto a la primera hipótesis de estudio, se ha podido comprobar la existencia de una diferencia significativa entre los dos tipos de colegios en orden a la frecuencia y tipología de discapacidad diagnosticada en los alumnos incluidos: los públicos acogen a un porcentaje ligeramente mayor de estos alumnos (5,51\%) con respecto a los concertados (4,66\%); además, los primeros incluyen a la mayoría de estudiantes $(72,2 \%)$ que presenta una discapacidad de grado ligero, una pequeña parte $(27,8 \%)$ de grado moderado y ningún alumno (0\%) de grado grave/completo, mientras que en los segundos la totalidad de los alumnos incluidos (100\%) presenta una discapacidad de grado ligero. Llama la atención el hecho de que en ninguno de los dos tipos de colegios cursan alumnos cuyo grado de diagnóstico sea severo-grave o completo. Estos datos se acercan a la situación de criticidad destacada en la introducción en orden a las dificultades persistentes para poder generalizar la inclusión en el ámbito educativo español, puesto que los datos recogidos sobre las dinámicas educativas inclusivas nos informan que, en España, aquellos estudiantes con alguna discapacidad que no pueden ser atendida en las clases ordinarias, siguen cursando sus estudios aisladamente fuera de las mismas (Canevaro y Malaguti, 2015; CERMI, 2010; Florian, 2014). Otra diferencia entre los dos tipos de centros educativos se refiere a los recursos existentes. Atendiendo a los recursos humanos, en términos de ratio profesor especialista/alumno con discapacidad, no se observa la misma proporción entre los dos centros educativos: en los públicos hay un profesor especialista cada tres alumnos con discapacidad, mientras que en los concertados uno cada cuatro alumnos. Esta diferencia se podría explicar teniendo en cuenta que en los colegios concertados no cursan 
alumnos con discapacidades de tipo grave-completo, ni moderado, con lo cual se requerirían menos profesores especialistas. Esta hipótesis se ve respaldada por los resultados obtenidos en un estudio desarrollado por el Ministerio Español de Educación, Cultura y Deporte (Martín et al., 2012). Por otro lado, atendiendo a los recursos materiales de apoyo a la docencia para atender a la diversidad, los colegios concertados obtienen una mayor puntuación en comparación con los públicos, como queda reflejado en la relación significativa, obtenida en nuestros análisis, entre el tipo de colegio y el factor de primer orden "recursos centro". Sorprende observar que en los centros públicos cursan más alumnos discapacitados y con discapacidades más graves que en los concertados y, a la vez, que el profesorado que trabaja en los centros públicos expresa insatisfacción respecto a los recursos y apoyos proporcionados. En cuanto a este supuesto, los resultados de los análisis correlacionales, que reflejan una relación estadísticamente significativa entre las variables de primer orden "recursos centro" y "actitudes favorables/desfavorables", nos llevan a establecer que la carencia de recursos disponibles influye positivamente en la resistencia del profesorado ante las prácticas inclusivas. Este supuesto queda confirmado, además, en el estudio de Tortello y Elgier (2014).

En segundo lugar, con respecto al segundo objetivo del estudio (comprobar la relación entre actitudes inclusivas y variables relativas al profesorado), la correlación positiva entre los factores "actitud favorable" e "inclusión positiva" indica precisamente que aquellos profesores que tienen una actitud favorable, presentan también una percepción positiva de la inclusión. En concreto, la percepción positiva hace referencia a la consideración de la inclusión como una propuesta educativa deseable que aporta efectos positivos, fomenta la comprensión y aceptación de las discapacidades, anula las diferencias y forma a todos los alumnos para una sociedad inclusiva. Estos resultados coinciden con los obtenidos en estudios anteriores (Martínez y Castillo, 2007; Mula et al., 2002). Lo mismo ocurre en la correlación encontrada entre los factores "actitud desfavorable" e "inclusión negativa aula ordinaria", que informa sobre aquellos profesores con actitudes desfavorables ante la inclusión que también tienen una percepción negativa de las perspectivas inclusivas: en este caso la percepción negativa se refiere específicamente al hecho de que el profesorado considera difícil mantener el orden y la disciplina en una clase ordinaria que acoge a alumnos con discapacidad, porque eso supone una atención extra que perjudicaría a los demás alumnos. En este aspecto los resultados coinciden con las investigaciones de Doménech, Esbrí, González, y Miret (2004) y de Arrebola y Giménez (2004).

Por otro lado, con respecto a otras variables del profesorado analizadas en este estudio, cabe destacar que el género, el nivel educativo y el tipo de centro en el que se desempeña la labor docente, no se revelaron variables predictoras de la postura general negativa del profesorado ante la inclusión, ya que no se superó el criterio de significación estadística, en acuerdo con los resultados de aquellos estudios que analizaron la influencia de estas variables (Clavijo, López, Cedillo, Mora, y Ortiz, 2016; Vélez, Tárraga, Fernández, y Sanz, 2017). No obstante, y a pesar de que existan resultados contradictorios con respecto a la variable edad (Ernst y Rogers, 2009; Forlin et al., 2009), Tortello y Elgier (2014) confirmaron que a medida que aumenta la edad se incrementan las actitudes desfavorables. Siguiendo esta última línea, en nuestro estudio se evidencia que la edad correlaciona negativamente con la postura general positiva.

Además, atendiendo a la segunda hipótesis del estudio, según la cual aquellos docentes con menos años de enseñanza apoyarían más la inclusión, los análisis de regresión lineal múltiple han podido confirmar una correlación positiva entre postura general negativa y años de docencia. Resultados afines a estos se han obtenido en estudios previos (Avramidis y Norwich, 2010; De Boer et al., 2011; Gónzalez-Gil et al., 2016), aunque cabe destacar que 
existen, también, resultados opuestos que informan que la postura negativa ante la inclusión no se ve condicionada por la cantidad de años que el profesorado lleva impartiendo clases (Avramidis et al., 2000; Dupoux, Hammond, Ingalls, y Wolman, 2006).

Por último, la tercera hipótesis de este estudio miraba a comprobar la existencia de una relación de proporcionalidad inversa entre postura general positiva del profesorado y creencia de que la responsabilidad sobre las adaptaciones curriculares sea exclusiva de los especialistas. A tal propósito, se ha podido confirmar la existencia de una relación positiva estadísticamente significativa entre postura inclusiva positiva y percepciones favorables acerca de los esfuerzos colaborativos y de las responsabilidades compartidas para atender a la discapacidad. Dicho con otras palabras, el principio de que los profesores de áreas se encarguen juntos con los demás especialistas, de la elaboración, puesta en práctica y seguimiento de las adaptaciones curriculares, determina en el profesorado una postura positiva ante la inclusión. Siguiendo esta línea, Lusk, Thompson, y Daane (2008) apoyarían nuestros resultados.

\section{Conclusiones}

Existe un general acuerdo en considerar que persisten dificultades para generalizar la inclusión en el ámbito educativo español en el que, los alumnos con discapacidades que no pueden ser atendidas adecuadamente en centros ordinarios, siguen acudiendo a institutos especiales (Canevaro y Malaguti, 2015; Florian, 2014).

Entre las criticidades ínsitas en los sistemas educativos inclusivos se destacan las actitudes negativas de los profesionales de la educación, que representan las barreras mentales que mayormente inciden en el proceso de inclusión socio-educativa de las personas con discapacidad (Echeíta, 2016; Flórez, León, y Alcedo, 2009; Martínez, 2012). Estas actitudes negativas de los profesores se deben principalmente a niveles inadecuados de recursos, formación y colaboración (Gandasegui y Méndez, 2014; De Boer et al., 2011).

Por otro lado, la presencia de apoyos, la competencia profesional, la capacidad de los profesionales de trabajar de forma conjunta, diseñando y realizando en equipo procesos de adaptación curricular, son elementos que constituyen la piedra angular de una inclusión exitosa (Torres y Fernández, 2015). Una colaboración profesional efectiva requiere un intercambio de experiencias y conocimientos entre especialistas y profesores de educación general, que conduciría al uso de estrategias educativas beneficiosas para cada alumno (con o sin discapacidad) y decisivas para el éxito de cualquier proceso educativo, especialmente el inclusivo (Carter y Hughes, 2005; McLesky y Waldron; 2002; Valeo, 2008).

Estos temas indican que es necesario garantizar la presencia de alumnos con discapacidad en cada centro ordinario para poder planificar concretamente los cambios que supone una educación verdaderamente inclusiva, en orden a procesos de organización escolar, recursos y competencias profesionales. Así mismo, se plantea la importancia de encargarse a nivel administrativo de formar activamente a todos los profesores, independientemente de sus especialidades, en comunicar entre ellos y saber proporcionar de forma colaborativa respuestas a cada necesidad que pueda surgir en un contexto educativo inclusivo. 


\section{Referencias}

Alemany, A.I., \& Villuendas, G.M.D. (2004). Las actitudes del profesorado hacia el alumnado con necesidades educativas especiales. Convergencia: Revista de Ciencias Sociales, 11(34), 183-215.

Alghazo, E.M., \& Gaad, E.N. (2004). General education teachers in the Unites Arab Emirates and their acceptance of the inclusion of students with disabilities. British Journal of Special Education, 31(2), 94-99. doi: https://doi.org/10.1111/j.0952-3383.2004.00335.x

Antonak, R.F., \& Larrivee, B. (1995). Psychometric analysis and revision of the Opinions Relative to Mainstreaming scale. Exceptional Children, 62(2), 139-149. doi: https://doi.org/10.1177/001440299506200204

Avramidis E., \& Norwich B. (2010). Teachers' attitudes towards integration/inclusion: A review of literature. European Journal of Special Needs Education, 17(2), 129-147. doi: https://doi.org/10.1080/08856250210129056

Avramidis, E., Bayliss, P., \& Burden, R. (2000). A survey into mainstream teachers' attitudes towards the inclusion of children with special educational needs in the ordinary school in one local education authority. Educational Psychology, 20(2), 191-211. doi: https://doi.org/10.1080/713663717

Batsiou, S., Bebetsos, E. Panteli, P. \& Antoniou, P. (2008). Attitudes and intention of greek and cypriot primary education teachers towards teaching pupils with special Education needs in mainstream schools. International Journal of Inclusive Education, 21 (2), 201-219.

Canevaro, A., \& Malaguti, E. (2015). Inclusione ed educazione: sfide contemporanee nel dibattito intorno alla pedagogia speciale. Italian Journal of Special Education for Inclusion, 2(2), 97-108. doi: https://doi.org/10.3280/es2014-003015

Carreres, L.A., \& Arnaiz, S.P. (2010). Evaluación de las prácticas educativas del profesorado de los centros escolares: indicadores de mejora desde la educación inclusiva. Revista Iberoamericana sobre Calidad, Eficacia y Cambio en Educación, 8(5), 96-109.

Carter, E.W., \& Hughes, C. (2005). Increasing social interaction among adolescents with intellectual disabilities and their general education peers: Effective interventions. Research and Practice for Persons with Severe Disabilities, 30, 179-193. doi: https://doi.org/10.2511/rpsd.30.4.179

Chiner, E. (2011). Las percepciones y actitudes del profesorado hacia la inclusión del alumnado con necesidades educativas especiales como indicadores del uso de prácticas educativas inclusivas en el aula (Tesis doctoral). Universidad de Alicante, España.

Clavijo, R., López, C., Cedillo, C., Mora, C., \& Ortiz, W. (2016). Actitudes docentes hacia la educación inclusiva en Cuenca. Maskana, 7(1), 13-22.

Comité Español de Representantes de Personas con Discapacidad (2010). Derechos humanos y discapacidad. Informe España 2010. Madrid: CERMI.

De Almeida, S.M., \& Castiñeiras, J.R.A. (2009). Las concepciones de los profesores y la respuesta a la inclusión en Lisboa. Revista de Educación Inclusiva, 2(2), 31-39.

De Boer, A., Pijil, S., \& Minnaert, A. (2011). Regular primary school teachers' attitudes towards inclusive education: a review of the literature. International Journal of Inclusive Education, 15(3), 331-353. doi: https://doi.org/10.1080/13603110903030089 
DeSimone, J.R., \& Parmar, R.S. (2006). Middle school mathematics teachers' beliefs about inclusion of students with learning disabilities. Learning Disabilities Research \& Practice, 21(2), 98-110. doi: https://doi.org/10.1111/j.1540-5826.2006.00210.x

Doménech, V., Esbrí, J.V., González, H.A., \& Miret, L. (2004). Actitudes del profesorado hacia el alumnado con Necesidades Educativas Especiales Derivadas de Discapacidad. Trabajo presentado en Novenes Jornades de Foment de la Investigació (Jornadas), Universitat Jaume-I, Castelló, España.

Domínguez-Lara, S. (2018). Fiabilidad y alfa ordinal. Actas Urológicas Españolas, 42(2), 140141. doi: 10.1016/j.acuro.2017.07.002

Dupoux, E., Hammond, H., Ingalls, L., \& Wolman, C. (2006). Teachers' Attitudes toward Students with Disabilities in Haiti. International Journal of Special Education, 21(3), 114. doi: https://doi.org/10.1177/875687050302200204

Dupoux, E., Wolman, C., \& Estrada, E. (2005). Teachers' attitudes toward integration of students with disabilities in Haiti and the United States. International Journal of Disability, Development, and Education, 52(1), 43-58. doi: https://doi.org/10.1080/10349120500071894

Echeíta, S.G. (2016). Inclusión y exclusión educativa. De nuevo, «Voz y quebranto». Revista Iberoamericana sobre Calidad, Eficacia y Cambio en Educación, 11(2), 99-118.

Elosua, P. \& Zumbo, B.D. (2008). Coeficientes de fiabilidad para escalas de respuesta categórica ordenada. Psicothema, 20(4), 896-901.

Ernst, C., \& Rogers, M.R. (2009). Development of the inclusion attitude scale for high school teachers. Journal of Applied School Psychology, 25, 305-322. doi: https://doi.org/10.1037/t44677-000

Flores, L., \& Gallego, L.V. (2015). Actitudes hacia la inclusión educativa de futuros maestros de inglés. Revista Latinoamericana de Educación Inclusiva, 9(1), 63-75.

Flórez, M. A., León, A. \& Alcedo, M.A. (2009).Revisión y análisis de los programas de cambio de actitudes hacia personas con discapacidad». Anuario de Psicología Clínica y de la Salud, 5, 85-98.

Florian, L. (2014). ¿What counts as evidence of inclusive education?. European Journal of Special Needs Education, 29(3), 286-294. doi: https://doi.org/10.1080/08856257.2014.933551

Forlin, C., Loreman, T., Sharma, U., \& Earle, C. (2009). Demographic differences in changing preservice teachers' attitudes, sentiments and concerns about inclusive education. International Journal of Inclusive Education, 13(2), 195-209. doi: https://doi.org/10.1080/13603110701365356

Gallego, J., \& Rodríguez, A. (2012). ¿Cómo aprenden ahora los estudiantes de magisterio a atender a la diversidad de su alumnado? Revista de Currículum y Formación del Profesorado, 16(2), 327-342.

Gandasegui, V.D., \& Méndez, F.C. (2014). Los recursos educativos que los profesores de Secundaria estiman necesarios para desarrollar procesos educativos inclusivos. Revista Española de Discapacidad, 2(1), 97-113. 
García, F., Inglés, C., Vicent, M., González, C., \& Mañas, C. (2013). Actitudes hacia la discapacidad en el ámbito educativo a través del SSCl (2000-2011). Análisis Temático y Bibliométrico. Electronic Journal of Research in Educational Psychology, 11(1), 139166. doi: https://doi.org/10.25115/ejrep.v11i29.1561

García, J.A. (2016). Actitudes hacia la discapacidad de aspirantes a educadores sociales. propuestas de formación para una educación inclusiva. Revista de Educación Inclusiva, 9(1), 13-29.

García, J.N., \& Alonso, J.C. (1985). Actitudes de los maestros hacia la integración escolar de niños con necesidades especiales. Infancia y Aprendizaje, 8(30), 51-68. doi: https://doi.org/10.1080/02103702.1985.10822070

Gómez, E. (2016). Actitudes de estudiantes de magisterio en educación primaria hacia las personas con discapacidad. Revista de Educación Inclusiva, 9(1), 54-69. doi: https://doi.org/10.1174/1135640042802455

Gónzalez-Gil, F., Martín-Pastor, E., Poy, R. \& Jenaro, C. (2016). Percepciones del profesorado sobre la inclusión: estudio preliminar. Revista Electrónica Interuniversitaria de Formación del Profesorado, 19(3), 11-24.

Hsien, M. (2007). Teacher Attitudes towards Preparation for Inclusion in Support of a Unified Teacher Preparation Program. Postgraduate Journal of Education Research, $8(1), 49-60$.

Hwang, Y., \& Evans, D. (2011). Attitudes towards inclusion: gaps between belief and practice. International Journal of Special Education, 26(1), 136-146.

Jordan A., Schwartz, E., \& McGhie, D. (2009). Preparing teachers for inclusive classrooms. Teaching and Teacher Education, 25(4), 535-542. doi: https://doi.org/10.1016/j.tate.2009.02.010

Kalyva, E., Gojkovic, D., \& Tsakiris, V. (2007). Serbian teachers' attitudes towards inclusion. International Journal of Special Education, 22(3), 30-35.

Kavale, K., \& Forness, S.R. (2000). History, rhetoric, and reality: Analysis of the inclusion debate. Remedial and Special Education, 21(5), 279-296. doi: https://doi.org/10.1177/074193250002100505

Koay, T., Lim, L., Sim, W., \& Elkins, J. (2006). Learning assistance and regular teachers' perceptions of inclusive education in Brunei Darussalam. International Journal of Special Education, 21(1), 131-142. doi: https://doi.org/10.1080/02188791.2004.10600205

Ley Orgánica 2/2006 de 3 de mayode Educación (LOE). Boletín Oficial del Estado (España), 4 de mayo de 2006.

Lorenzo-Seva, U., \& Ferrando, P.J. (2006). FACTOR: A computer program to fit the exploratory factor analysis model. Behavior Research Methods, 38(1), 88-91. doi: 10.3758/BF03192753.

Lusk, A., Thompson, T., \& Daane, C.J. (2008) Algebra 1 teachers' perceptions of teaching students with learning disabilities. Journal of Curriculum and Instruction, 2(2), 34-51. doi: https://doi.org/10.3776/joci.2008.v2n2p34-51

Marchesi, Á., Durán, D., Giné, C., \& Hernández, L. (2009). Guía para la reflexión y valoración de prácticas inclusivas. Madrid: Organización de Estados Iberoamericanos para la Educación, la Ciencia y la Cultura. 
Martín, M., Ferreira, M., Velázquez, E.D., Enríquez, M.F., Fernández, N.V., \& De Esteban, C.G. (2012). Sobre la educación inclusiva en España: políticas y prácticas. Intersticios: Revista Sociológica de Pensamiento Crítico, 6(1), 279-295.

Martínez S.R. (2012). Discapacidad e integración educativa: ¿qué opina el profesorado sobre la inclusión de estudiantes con discapacidad en sus clases? Revista Española de Orientación y Psicopedagogía, 23 (3), 96-109.

Martínez, L.N., \& Castillo, A.I. (2007). Actitudes del profesorado de conservatorio sobre la integración educativa: un análisis exploratorio. Revista Española de Orientación y Psicopedagogía, 18(1), 47-57.

McLesky, J., \& Waldron, N.L. (2002). Inclusion and school change: Teacher perceptions regarding curricular and instructional adaptations. Teacher Education and Special Education, 25(1), 41-54. doi: https://doi.org/10.1177/088840640202500106

Moya M.A., \& Díez, S.U. (2012). La evaluación estatal del Plan de Refuerzo, Orientación y Apoyo (PROA). Análisis tras seis años de evaluación continuada. Revista de Educación, (1), 89-116.

Mula, A., Alaminos, A., Navas, L., Torregrosa, G., Sabroso, A., López-Cano, D., ... \& Santacreu, O. (2002). Incidencia de las actitudes y expectativas de alumnos y profesores sobre el desarrollo del programa de integración. Alicante, España: Universidad de Alicante.

Piccioli, M. (2019). Il processo italiano di inclusione scolastica nella prospettiva internazionale: i Disability Studies come sviluppo inclusivo. Formazione, lavoro, persona, 20, 91-99.

Rajakaltio, H., \& Mäkinen, M. (2014). The Finnish school in cross-pressures of change. European Journal of Curriculum Studies, 1(2), 133-140.

Sales, C.A., Moliner, G.O., \& Sanchiz, R.M.L. (2001). Actitudes hacia la atención a la diversidad en la formación inicial del profesorado. Revista Electrónica Interuniversitaria de Formación del Profesorado, 4(2), 1-7.

Sandoval, M.M., Simón, R.C., \& Echeíta, S.G. (2012). Análisis y valoración crítica de las funciones del profesorado de apoyo desde la educación inclusiva. Revista de Educación, (Número extr.), 117-137.

Sharma U., Forlin C., Loreman T., \& Earle, C. (2006). Pre-service teachers' attitudes, concerns and sentiments about inclusive education: An international comparison of the novice pre-service teacher. International Journal of Special Education, 21(2), 8093. doi: https://doi.org/10.1080/13603110701365356

Sharma, U., Moore, D., \& Sonawane, S. (2009). Attitudes and concerns of preservice teachers regarding inclusion of students with disabilities into regular schools in Pune, India. Asia-Pacific Journal of Teacher Education, 37(3), 319-331. doi: https://doi.org/10.1080/13598660903050328

Smith, A. (2004). The inclusion of pupils with special educational needs in secondary school physical education. Physical Education and Sport Pedagogy, 9(1), 27-43. doi: https://doi.org/10.1080/1740898042000208115

Tam, B.K., Seevers, R., Gardner, R., \& Heng, M.A. (2006). Primary school teachers' concerns about the integration of students with special needs in Singapore. Teaching Exceptional Children Plus, 3(2), 78-96. 
Tárraga, R., Grau, C., \& Peirat, J. (2013). Actitudes de los estudiantes del Grado de Magisterio y del Máster de Educación Especial hacia la inclusión educativa. Revista Electrónica Interuniversitaria de Formación del Profesorado, 16(1), 55-72. doi: https://doi.org/10.6018/reifop.16.1.179441

Torres, J.A. \& Fernández, J.M. (2015). Promoviendo escuelas inclusivas: análisis de las percepciones y necesidades del profesorado desde una perspectiva organizativa, curricular y de desarrollo profesional. Revista Electrónica Interuniversitaria de Formación del Profesorado, 18 (1), 177-200. doi: http://dx.doi.org/10.6018/reifop.18.1.214391

Tortello, C., \& Elgier, Á.M. (2014). Integración escolar en Argentina: un estudio preliminar. Psicología Educativa, 2, 33-41.

Valeo, A. (2008). Inclusive education support Systems: Teacher and administrator views. International Journal of Special Education, 23(2), 8-16.

Van Reusen, A.K., Soho, A.R., \& Barker, K.S. (2001). High school teacher attitudes toward inclusion. High School Journal, 84(2), 7-21.

Vélez, C.X., Tárraga, M.R., Fernández, A.M.I., \& Sanz, C.P. (2017). Formación inicial de maestros en Educación Inclusiva: una comparación entre Ecuador y España. Revista de Educación Inclusiva, 9(3), 75-94.

Wilkins, T., \& Nietfeld, J.L. (2004). The effect of a school-wide inclusion training programme upon teachers' attitudes about inclusion. Journal of Research in Special Educational Needs, 4(3), 115-121. doi: https://doi.org/10.1111/j.1471-3802.2004.00026.x 